\title{
Light Injection in SOI Microwaveguides Using High-Efficiency Grating Couplers
}

\author{
Laurent Vivien, Daniel Pascal, Sebastien Lardenois, Delphine Marris-Morini, Eric Cassan, \\ Frédéric Grillot, Associate Member, IEEE, Suzanne Laval, Jean-Marc Fédéli, and Loubna El Melhaoui
}

\begin{abstract}
An experimental characterization of the grating couplers for sub-micrometer silicon-on-insulator (SOI) waveguides is presented. The grating couplers have been designed, realized, and characterized for the +1 diffraction order at an operating wavelength of $1.31 \mu \mathrm{m}$ for TE polarization. At the resonant angle, a coupling efficiency higher than $55 \%$ has been measured. The angular coupling range and the wavelength tolerance have been evaluated to $3^{\circ}$ and $20 \mathrm{~nm}$, respectively. The grating coupler is followed by a taper, and about $50 \%$ of the input power at $1.31 \mu \mathrm{m}$ is coupled into sub-micrometer rib and strip SOI waveguides. The ration between light power decoupled toward the cladding and light power decoupled toward the substrate is about three.
\end{abstract}

Index Terms-Grating coupler, optical coupling, rib waveguide, silicon-on-insulator (SOI).

\section{INTRODUCTION}

$\mathbf{S}$ ILICON-ON-INSULATOR (SOI) substrate shows great potential for the fabrication of low-cost photonic integrated optical circuits and optoelectronic devices such as optical modulators [1], [2], low loss optical waveguides [3]-[7], and photodetectors [8], [9]. SOI substrate is an attractive platform as it allows the miniaturization of waveguide cross-sectional area to sub-micrometer scales due to the high refractive index contrast between silicon and its oxide $(\Delta n \sim 2)$. One of the main advantages of the silicon technology is its full compatibility with CMOS technology. SOI substrate is of prime importance for integrated optoelectronic circuits with the potential monolithic integration of optical and electronic functions on a single chip.

SOI is commercialized with buried oxide thickness typically ranging from $50 \mathrm{~nm}$ to $3 \mu \mathrm{m}$ and silicon film thickness from a few tens of nanometers to $1.5 \mu \mathrm{m}$ that can be well suited to optical applications [10]. The thin upper silicon film is used as an optical waveguide at telecommunication wavelengths larger

Manuscript received December 15, 2005; revised April 20, 2006. This work was supported by the French RMNT projects "INOPCIS" and "CAURICO" in the frame of collaboration between Institut d'Électronique Fondamentale (CNRS/UPS Orsay), CEA-LETI (Grenoble), Laboratoire de Physique de la Matière (INSA Lyon), CNAM (Paris), and ST Microelectronics (Crolles).

L. Vivien, D. Pascal, D. Marris-Morini, E. Cassan, and S. Laval are with the Institut d'Electronique Fondamentale, Universite Paris-Sud, 91405 Orsay Cedex, France (e-mail: vivien @ief.u-psud.fr).

S. Lardenois was with the Institut d'Electronique Fondamentale, Universite Paris-Sud, 91405 Orsay Cedex, France. He is now with CEA/LETI, 38054 Grenoble Cedex 9, France.

F. Grillot was with the Institut d'Electronique Fondamentale, Universite Paris-Sud, 91405 Orsay Cedex, France. He is now with the Laboratoire d'Etudes des Nanostructures a Semiconducteurs, Institut National des Sciences Appliquees, 67084 Strasbourgh Cedex, France.

J.-M. Fédéli and L. El Melhaoui are with CEA/LETI, 38054 Grenoble Cedex 9, France.

Digital Object Identifier 10.1109/JLT.2006.878060 than $1.2 \mu \mathrm{m}$, for which silicon is transparent. The buried silicon oxide (BOX) thickness must be higher than $700 \mathrm{~nm}$ (respectively $1 \mu \mathrm{m}$ ) to ensure negligible light leakage toward the $\mathrm{Si}$ substrate at a wavelength of $1.3 \mu \mathrm{m}$ (respectively $1.55 \mu \mathrm{m}$ ).

One of the main forecast applications is high-frequency clock signal distribution in microelectronic integrated circuits [11]. The used wavelength $\lambda=1.3 \mu \mathrm{m}$ and TE polarization are advantageously chosen for system compactness. The lateral field confinement of the guided light can be obtained either by partial etching or by complete etching of the silicon film to define either rib or strip SOI waveguides. The optical propagation losses are a few decibels per centimeter for strip waveguides [4]-[6] and can be as small as $0.1 \mathrm{~dB} / \mathrm{cm}$ for rib sub-micrometer ones [7]. Compactness of the photonic devices leads to increase light coupling difficulties into sub-micrometer structures and became crucial for integrated devices that present transmittance drops of a few tens of decibels like optical distributions to several outputs [12], [13]. Thus, it is very important to reduce all extra loss due to light injection into the input waveguide 1) to allow silicon nanophotonic development and 2) to allow accurate characterizations.

The commonly used solution to couple light into a submicrometer waveguide is to directly focus the laser beam from a lensed fiber onto the end of the sub-micrometer waveguide at the chip edge (butt coupling). Even with guides enlarged near the facet to a few micrometers, this technique introduces coupling losses typically higher than $12 \mathrm{~dB}$.

Nanotapers for compact-mode conversion to couple light from an optical fiber to a sub-micrometer waveguide at $1.55 \mu \mathrm{m}$ have been demonstrated without [14] and with polymer waveguide on the silicon tip [15]. A high accuracy is required for the fabrication of the nanometer-sized tip as well as for the realization of the following cleaving step. A polarizationinsensitive three-dimensional (3-D) taper that reduces these limitations has been proposed [16].

Another solution is to use grating couplers followed by a taper to adjust the lateral size of the incident beam to the sub-micrometer waveguide width. The main advantage of a grating coupler is the possible use of test devices everywhere on the die without any cleavage. Several grating couplers have been demonstrated theoretically [17], [18] and experimentally [19]-[21]. However, the highest coupling efficiency reported in sub-micrometer rib and strip SOI waveguides does not exceed $40 \%$ [22].

This paper reports optimized one-dimensional (1-D) grating couplers that present coupling efficiency into sub-micrometer waveguides higher than $55 \%$. Couplers have been designed, 
(a)

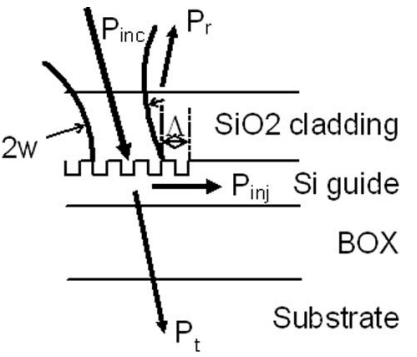

(b)

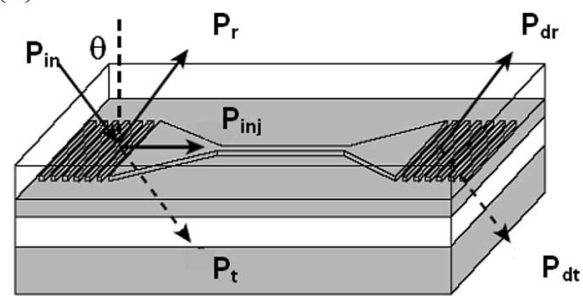

Fig. 1. (a) Schematic view of the grating coupler lightened by a Gaussian beam. $P_{\text {in }}$ is the input power, and $P_{t}$ and $P_{r}$ are, respectively, the transmitted and reflected powers. $P_{\text {inj }}$ is the injected power below the grating coupler. $w$ is the waist of the Gaussian beam. $\Lambda$ is the period of the grating coupler. (b) Example of test device for guided structure characterization. The waveguide is inserted between two grating couplers with linear transitions. $P_{\mathrm{dt}}$ and $P_{\mathrm{dr}}$ are, respectively, the transmitted and reflected output powers.

realized, and characterized at $\lambda=1.3 \mu \mathrm{m}$ and used to determine optical propagation losses in sub-micrometer strip and rib SOI devices. The significant improvements carried out to increase the coupling efficiency using grating couplers are, in the first hand, the optimization of fabrication technology leading to a better accuracy of all layer thicknesses, period, and etching depth, and in the second hand, the optimization of illumination conditions (input beam diameter optimization). Section II reports the numerical simulation results of the grating coupler efficiency. The technological realization is described in Section III. In the last section, experimental results about coupling efficiency, resonant angle, and wavelength dependence are presented for two waveguide geometries.

\section{NumeriCAL SimUlations}

The simulated structure is schematically depicted in Fig. 1(a). It consists of an SOI substrate covered with a 700-nmthick $\mathrm{SiO}_{2}$ cladding layer. The coupling efficiency strongly depends on the thickness of both the silicon film $(n=3.505)$ and the $\operatorname{BOX}(n=1.45)$. The mode is resonantly excited when the phase matching condition is fulfilled, i.e.,

$$
k_{\text {in }} \sin \left(\theta_{\text {in }}\right)+p \frac{2 \pi}{\Lambda}=\beta^{\prime}
$$

where $k_{\mathrm{in}}=2 \pi / \lambda$ is the module of the incident wave vector, $\lambda$ is the wavelength, $\theta_{\text {in }}$ is the incident angle, $p$ is the diffraction order (here $p=+1), \Lambda$ is the grating period, $\beta^{\prime}=(2 \pi / \lambda) n_{\mathrm{eff}}$ is the real part of the propagation constant, and $n_{\text {eff }}$ is the effective index of the guided mode. The calculations are based on a differential analysis [23] allowing the determination of the optical field under the grating for the input plane wave case. With this plane wave approach, the power attenuation

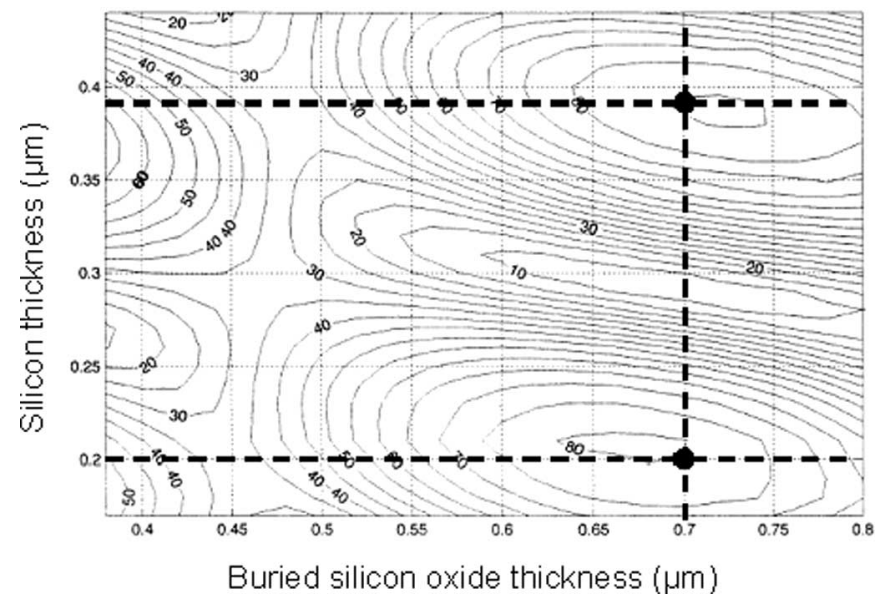

Fig. 2. Coupling efficiency as a function of silicon waveguide and BOX thicknesses. The period is $430 \mathrm{~nm}$, and the etching depth $30 \mathrm{~nm}$, without silica cladding.

coefficient $\left(1 / L_{c}\right)$ under the grating (or grating characteristic length $\left.L_{c}\right)$ is

$$
L_{c}=\frac{\lambda}{4 \pi n_{\mathrm{eff}}^{\prime \prime}}
$$

where $n_{\text {eff }}^{\prime \prime}$ is the imaginary part of the effective index of the mode.

The geometrical parameters of the grating, thickness of the BOX, crystalline silicon, $\mathrm{SiO}_{2}$ cladding thicknesses, and etched depth are determined for optimum light coupling. Furthermore, this 1-D model allows the determination of the optimal laser beam waist that has to be used for the given grating coupler parameters

$$
\omega_{0}=1.37 L_{c} \cos \left(\theta_{\text {in }}\right)
$$

and of the optimal distance $d$ between the center of the input laser beam and the end of the grating coupler (beginning of the waveguide)

$$
d=L_{c} .
$$

Then, the use of a beam propagation method (BPM), including forced excitation, allows the characterization of the diffracted field (i.e., the guided mode) propagating in the corrugated waveguide for a 3-D Gaussian incident beam illuminating the grating. The module and direction of the incident wave vector are taken into account on each point of the device. At the end of the grating coupler, the field values are added up over the entire cross section of the guide. The division by the incident power gives the coupling efficiency [18]. Fig. 2 shows the coupling efficiency versus buried oxide silicon and silicon film thicknesses for a given grating coupler. The period is $430 \mathrm{~nm}$, and the etching depth is $30 \mathrm{~nm}$. The coupling efficiency strongly depends on both the silicon film and the buried oxide thicknesses. The maximum efficiency is the convolution between the Gaussian and the decoupled profile. Eighty-two percent is achieved for an exponential profile. Nevertheless, when $L_{c}$ is 

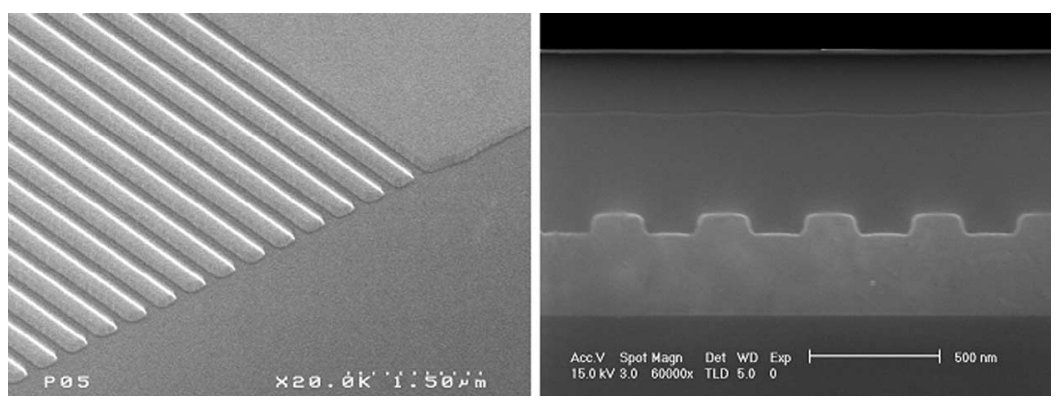

Fig. 3. SEM top and side views of an example of grating coupler.

small enough (about $10 \mu \mathrm{m}$ ), the decoupled power profile is not quite exponential, and its convolution with a Gaussian profile allows coupling efficiencies slightly higher than $82 \%$ [24]. For a 700-nm-thick buried oxide, a coupling efficiency close to $85 \%$ can be theoretically achieved for both 200- and 380-nm-thick silicon films.

According to the characteristic length (10-20 $\mu \mathrm{m})$, the grating coupler length must be large enough (typically $100 \mu \mathrm{m}$ ) in order to ensure that all the power is decoupled.

\section{REALIZATION}

The integrated devices have been fabricated on specific Unibond 200-mm wafers provided by SOITEC, Grenoble, France, fabricated by the smart cut process [10]. The crystalline silicon and the BOX thicknesses are 400 and $700 \mathrm{~nm}$, respectively. Two waveguide heights are used for this study: $380 \mathrm{~nm}$ (the initial silicon thickness slightly reduced during the process) and $200 \mathrm{~nm}$ (obtained by thermal oxidation). The grating couplers are defined using e-beam lithography. The global size is $30 \mu \mathrm{m}$ wide and $100 \mu \mathrm{m}$ long. The grating period is 430 and $500 \mathrm{~nm}$ for 380- and 200-nm waveguide thicknesses, respectively. The etching depths of the grating coupler groves are 70 and $30 \mathrm{~nm}$ for the 380- and 200-nm waveguide thicknesses, respectively. Etching is performed by a reactive ion etching (RIE) process. Thermal oxidation is performed to reduce the surface roughness, and a 700-nm-thick silicon oxide cladding layer is then deposited. Fig. 3 shows the top and side views of the grating coupler obtained with a scanning electron microscope (SEM). The waveguide height and the groove depth are 380 and $70 \mathrm{~nm}$, respectively. The darker region below the silicon grating is the 700-nm-thick buried $\mathrm{SiO}_{2}$ layer. At the end of the process, an antireflection coating for $\lambda=1.31 \mu \mathrm{m}$ is added on the polished back side of the silicon substrate in order to correctly determine the laser beam intensities reflected and transmitted by the grating.

\section{EXPERIMENTAL RESUlTS}

In order to measure coupling efficiencies and integrated structure characteristics, sub-micrometer SOI rib or strip devices are inserted between input and output grating couplers [Fig. 1(b)]. A 1-mm linear transition from the 30- $\mu \mathrm{m}$ width of the grating coupler to sub-micrometer SOI waveguide is used. This transition ensures reduced insertion losses for

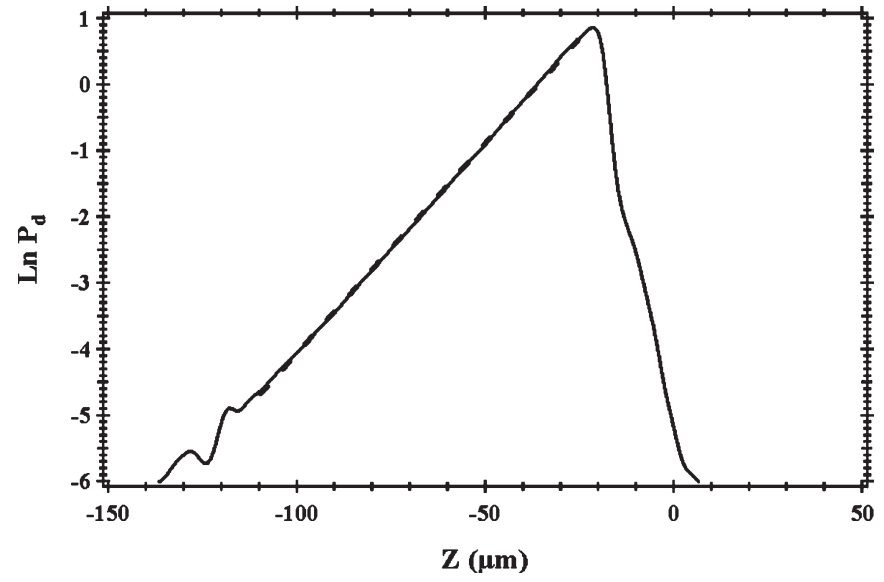

Fig. 4. Decoupled power of a grating coupler as a function of the distance on the grating for the 200-nm-thick rib SOI waveguide. The dotted curve is the fit by a linear curve allowing the determination of the coupling length.

slightly etched rib structures. The power $P_{\text {inj }}$ injected into the waveguide under the grating is

$$
P_{\text {inj }}=P_{\text {in }}-\left(P_{r}+P_{t}\right)
$$

where $P_{\text {in }}$ is the input power from the laser source at $1.31 \mu \mathrm{m}$, while $P_{r}$ and $P_{t}$ are the power fractions reflected and transmitted by the grating coupler, respectively. The coupling efficiency is then given by $P_{\mathrm{inj}} / P_{\mathrm{in}}$. The guided wave is decoupled by the output grating on both sides of the waveguide. The total light power decoupled at the waveguide output is equal to $P_{d}=$ $P_{\mathrm{dr}}+P_{\mathrm{dt}}$, where $P_{\mathrm{dr}}$ and $P_{\mathrm{dt}}$ are the parts of the decoupled power toward cladding and substrate, respectively. Using a $100-\mu \mathrm{m}$-long grating coupler, all of the power is decoupled either on the transmitted side $\left(P_{\mathrm{dt}}\right)$ or the reflected side $\left(P_{\mathrm{dr}}\right)$. Light power is measured on each of the four beams for a given incident intensity $P_{\text {in }}$ [Fig. 1(b)].

In order to determine the grating characteristics, the coupling length $\left(L_{c}\right)$ is first measured with a linear infrared camera. The decoupled power is proportional to $\exp \left(-z / L_{c}\right)$. Fig. 4 shows the decoupled power as a function of the distance on the grating for a 200-nm-thick waveguide. The obtained value for $L_{c}$ is equal to $13 \pm 1 \mu \mathrm{m}$. $L_{c}$ is directly linked to the laser beam waist $(\omega)$, which has to be used to obtain the maximum grating coupler efficiency (3). The corresponding waist thus ranges from 16 to $19 \mu \mathrm{m}$. By considering the grating size $(30 \mu \mathrm{m}$ wide and $100 \mu \mathrm{m}$ long), a waist of $16 \mu \mathrm{m}$ has been chosen. 


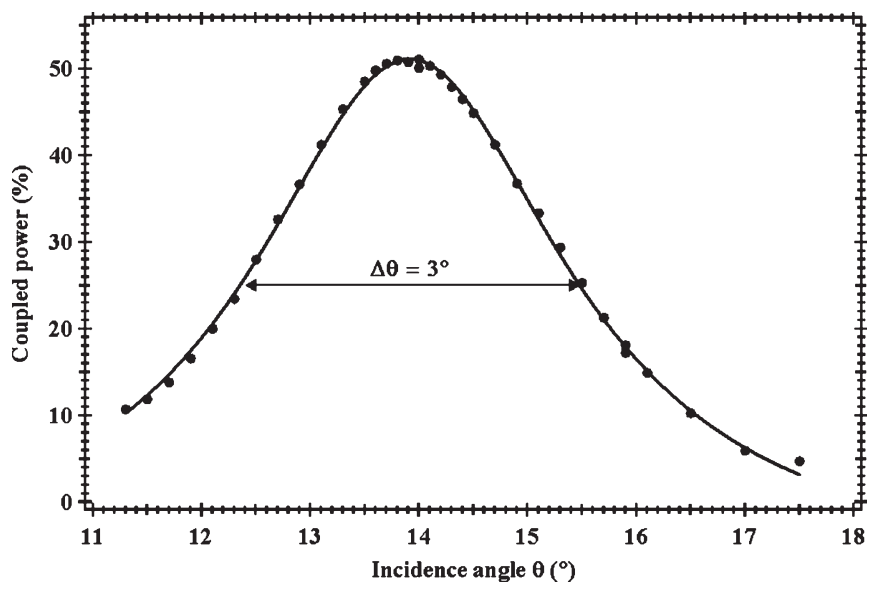

Fig. 5. Coupled power as a function of the incident angle for the 200-nm-thick rib SOI waveguide.

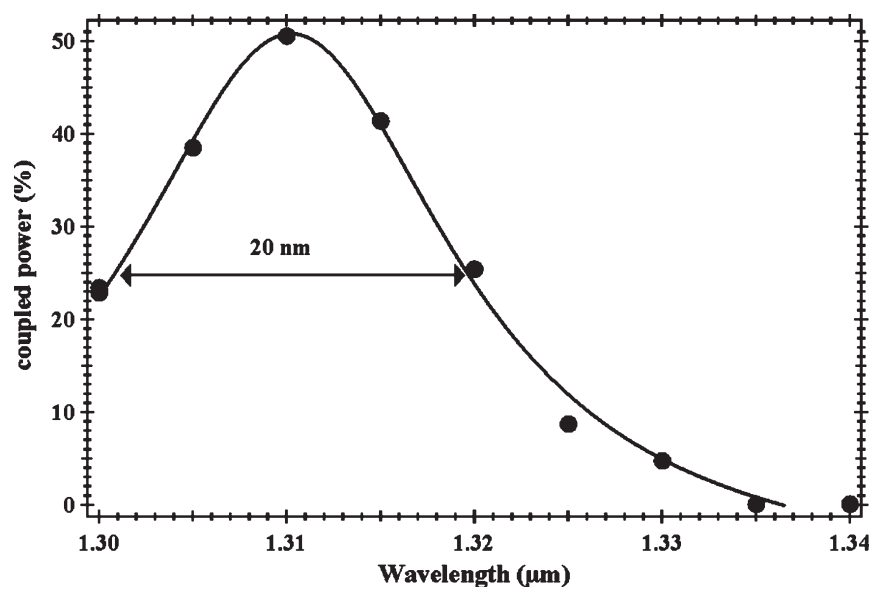

Fig. 6. Coupled power as a function of wavelength for the 200-nm-thick rib SOI waveguide.

The injected power $P_{\text {inj }}=P_{\text {in }}-\left(P_{R}+P_{T}\right)$ below the grating coupler as a function of the incidence angle of the laser beam is plotted in Fig. 5 for the 200-nm-thick SOI waveguide. The measured optimum incidence angle $(\theta)$ is $13.9^{\circ} \pm 0.2^{\circ}$. This corresponds to a maximum of the coupled power in a 200-nm-thick SOI waveguide of about 56\%. The width at half maximum of the angular response is then $3^{\circ}$, which corresponds to the angle tolerance range of the grating coupler. Similar results have been obtained for 380-nm-thick SOI waveguides with coupling efficiencies close to $60 \%$. In the case of a plane wave simulation, the angular coupling range is given by $\lambda / 4 \pi L_{c} \cos (\theta)$ and is about $0.5^{\circ}$. The difference between measured and calculated values is due to the input Gaussian beam divergence. Indeed, the measurements correspond to the convolution between the Lorentzian profile characterizing the grating coupler and the angular Gaussian repartition of the plane wave decomposition. For small waist spots, the angular acceptance of the coupling is given by the Gaussian beam divergence.

Coupling efficiency is plotted in Fig. 6 as a function of wavelength. The optimum wavelength that corresponds to the maximum coupling efficiency is $1.31 \mu \mathrm{m}$, which is the wavelength aimed at in the grating coupler design. The wave- length acceptance (measured at half maximum) is close to $20 \mathrm{~nm}$. Sub-micrometer SOI devices could be tested from 1.3 to $1.32 \mu \mathrm{m}$ with a coupling efficiency higher than $25 \%$.

To characterize sub-micrometer rib and strip SOI waveguides using grating couplers, tapers are used at the input and output. They enable to reduce the light beamwidth from the grating size $(30 \mu \mathrm{m})$ to a waveguide width of $1 \mu \mathrm{m}$ or $380 \mathrm{~nm}$ for rib or strip waveguides, respectively. One-millimeter-long linear transitions are used in order to carry out mode converter. Using field mode matching simulations, ${ }^{1}$ transition losses from grating to waveguides have been estimated. Theoretically, $95 \%$ of the power injected in the taper can be coupled into rib SOI structures ( $1 \mu \mathrm{m}$ wide), while about $75 \%$ is coupled into strip structures ( $380 \mathrm{~nm}$ wide). Mode conversions are observed at the end of the taper, leading to the decrease of the output power into single-mode waveguides. Experimentally, the measured taper transmissions are $\sim 92 \%$ for rib SOI waveguides and $\sim 62 \%$ for strip ones [7].

The decoupled power proportion toward the cladding is defined as $\eta=P_{\mathrm{dr}} /\left(P_{\mathrm{dr}}+P_{\mathrm{dt}}\right)$. For both 200- and 380-nm SOI waveguide thicknesses, $\eta$ is of about $75 \%$.

\section{CONCLUSION}

A high-efficiency grating coupler used to measure optical loss in sub-micrometer SOI devices for TE polarization at $1.31-\mu \mathrm{m}$ wavelength has been presented. Coupling length $\left(L_{c}\right)$ has been measured to determine the optimum beam waist to be used in the experimental setup. Experimentally, coupling efficiencies close to $55 \%$ to $60 \%$ for both 200 - and $380-\mathrm{nm}$ waveguide thicknesses have been measured. The angular alignment tolerance of $3^{\circ}$ is mainly related to the laser beam divergence. The wavelength tolerance is $20 \mathrm{~nm}$ at half maximum. By using tapers from grating couplers to sub-micrometer SOI waveguides, a coupled power higher than $50 \%$ is achieved in sub-micrometer rib and strip waveguides. Furthermore, the measured decoupled power proportion toward the cladding is close to $75 \%$, leading to carry out efficient coupling toward the optical fiber.

\section{ACKNOWLEDGMENT}

The authors would like to thank P. Cogez, V. Le Goascoz, and J. Torres of STMicroelectronics for fruitful discussions and the staff of the 200-mm cleanroom of LETI for the fabrication of high-quality optical structures.

\section{REFERENCES}

[1] D. Marris, A. Cordat, D. Pascal, A. Koster, E. Cassan, L. Vivien, and S. Laval, "Design of a SiGe-Si quantum well optical modulator," IEEE J. Sel. Topics Quantum Electron., vol. 9, no. 3, pp. 747-754, May/Jun. 2003.

[2] C. A. Barrios, V. R. Almeida, R. Panepucci, and M. Lipson, "Electrooptic modulation of silicon-on-insulator submicrometer-size waveguide devices," J. Lightw. Technol., vol. 21, no. 10, pp. 2332-2339, Oct. 2003.

[3] K. K. Lee, D. R. Lim, H.-C. Luan, A. Agarwal, J. Foresi, and L. C. Kimerling, "Effect of size and roughness on light transmission in a

${ }^{1}$ Oxford, U.K., Data are available at Photon Design, 34 Leopold Street, OX41TW. [Online]. Available: www.photond.com. 
$\mathrm{Si} / \mathrm{SiO}_{2}$ waveguide: Experiments and model," Appl. Phys. Lett., vol. 77, no. 1, pp. 1617-1619, Sep. 2000.

[4] K. K. Lee, D. R. Lim, and L. C. Kimerling, "Fabrication of ultralow-loss $\mathrm{Si} / \mathrm{SiO}_{2}$ waveguides by roughness reduction," Opt. Lett., vol. 26, no. 23, pp. 1888-1890, 2001.

[5] Y. A. Vlasov and S. J. McNab, "Losses in single-mode silicon-oninsulator strip waveguides and bends," Opt. Exp., vol. 12, no. 8, pp. 1622$1631,2004$.

[6] P. Dumon, W. Bogaerts, V. Wiaux, J. Wouters, S. Beckx, J. Van Campenhout, D. Taillaert, B. Luyssaert, P. Bienstman, D. Van Thourhout, and R. Baets, "Low-loss SOI photonic wires and ring resonators fabricated with deep UV lithography," IEEE Photon. Technol. Lett, vol. 16, no. 5, pp. 1328-1330, May 2004.

[7] S. Lardenois, D. Pascal, L. Vivien, E. Cassan, S. Laval, R. Orobtchouk, M. Heitzmann, N. Bouaida, and L. Mollard, "Low-loss sub-micrometer SOI rib waveguides and corner mirrors," Opt. Lett., vol. 28, no. 13, pp. 1150-1152, Jul. 2003.

[8] L. Fama, L. Colace, G. Masini, G. Assanto, and H. C. Luan, "High performance germanium-on-silicon detectors for optical communications," Appl. Phys. Lett., vol. 81, no. 4, pp. 586-588, Jul. 2002.

[9] L. Naval, B. Jalali, L. Gomelsky, and J. M. Liu, "Optimization of $\mathrm{Si}_{1-\mathrm{x}} \mathrm{Ge}_{\mathrm{x}} / \mathrm{Si}$ waveguide photodetectors operating at $\lambda=1.3 \mu \mathrm{m}$," J. Lightw. Technol., vol. 14, no. 5, pp. 787-797, May 1996.

[10] Data on silicon on insulator substrate are available at [Online]. Available: http://www.soitec.com

[11] International Technology Roadmap for Semiconductor: Interconnect. [Online]. Available: http://www.itrs.net/Links/2005ITRS/ Interconnect2005.pdf, pp. 1-63, 2005

[12] T. Fukazawa, A. Sakai, and T. Baba, "H-tree-type optical clock signal distribution circuit using a Si photonic wire waveguide," Jpn. J. Appl. Phys., vol. 41, no. 12B, pp. L1461-L1463, Dec. 2002.

[13] L. Vivien, S. Lardenois, D. Pascal, S. Laval, E. Cassan, J. L. Cercus, X. Le Roux, A. Koster, J. M. Fédéli, and M. Heitzmann, "Experimental demonstration of a low-loss optical $H$-tree distribution using silicon-oninsulator microwaveguides," Appl. Phys. Lett., vol. 85, no. 5, pp. 701-703, Aug. 2004.

[14] V. R. Almeida, R. R. Panepucci, and M. Lipson, "Nanotaper for compact mode conversion," Opt. Let., vol. 28, no. 1, pp. 1302-1304, Aug. 2003

[15] T. Tsuchizawa, K. Yamada, H. Fukuda, T. Watanabe, J. Takahashi, M. Takahashi, T. Shoji, E. Tamechika, S. Itabashi, and H. Morita, "Microphotonics devices based on silicon micro-fabrication technology," IEEE J. Sel. Topics Quantum Electron., vol. 11, no. 1, pp. 232-240, Jan./Feb. 2005.

[16] L. Vivien, S. Laval, E. Cassan, X. Le Roux, and D. Pascal, "2-D taper for low-loss coupling between polarization-insensitive microwaveguides and single-mode optical fibers," J. Lightw. Technol., vol. 21, no. 10, pp. 2429 2433, Oct. 2003.

[17] R. M. Emmons and D. G. Hall, "Buried-oxide silicon-on-insulator structures II: Waveguide grating couplers," IEEE J. Quantum Electron., vol. 28 , no. 1 , pp. 164-175, Jan. 1992.

[18] N. Landru, D. Pascal, and A. Koster, "Modelling of two-dimensional grating couplers on silicon-on-insulator waveguides using beam propagation method," Opt. Comm., vol. 196, no. 1-6, pp. 139-147, Sep. 2001.

[19] D. Taillaert, W. Bogaert, P. Bienstman, T. F. Krauss, P. Van Daele, I. Moerman, S. Verstuyft, K. De Mesel, and R. Baets, "An out-ofplane grating coupler for efficient butt-coupling between compact planar waveguides and single-mode fibers," IEEE J. Quantum Electron., vol. 38, no. 7, pp. 949-955, Jul. 2002.

[20] R. Orobtchouk, A. Layadi, H. Gualous, D. Pascal, A. Koster, and S. Laval, "High-efficiency light coupling in a submicrometric siliconon-insulator waveguide," Appl. Opt., vol. 39, no. 31, pp. 5773-5777, Nov. 2000.

[21] D. Pascal, R. Orobtchouk, A. Layadi, A. Koster, and S. Laval, "Optimized coupling of a Gaussian beam into an optical waveguide with a grating coupler: Comparison of experimental and theoretical results," Appl. Opt., vol. 36, no. 12, pp. 2443-2447, Apr. 1997.

[22] D. Pascal, S. Lardenois, E. Cassan, A. Koster, S. Laval, M. Heitzmann, L. Mollard, B. Dal'zotto, N. Bouzaïda, and R. Orobtchouk, "Efficient light coupling into sub-micrometer rib and strip SOI waveguides," in Proc. Integrated Photon. Res. 2002, Vancouver, BC, Canada, Jul. 17-19, 2002, p. ITh17-1.

[23] K. C. Chang, V. Shah, and T. Tamir, "Scattering and guiding of waves by dielectric gratings with arbitrary profiles," J. Opt. Soc. Amer. A, vol. 7, no. 7, pp. 804-812, Jul. 1980.

[24] S. Miyanaga and T. Asagura, "Intensity profile of outgoing beams from uniform and linearly tapered grating couplers," Appl. Opt., vol. 20, no. 4, pp. 688-695, Feb. 1981

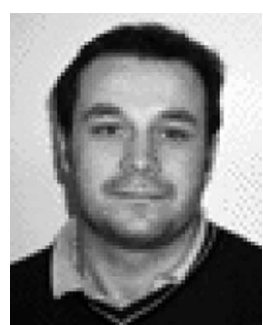

Laurent Vivien received the Ph.D. degree in physics from the Polytechnique School, Palaiseau, France, in 2001. His Ph.D. dissertation was on nonlinear optical properties of carbon nanotubes for optical limiting.

Between 2001 and 2003, he held a postdoctoral position with the Institute of Fundamental Electronics (IEF), Orsay, France, where he studied single-mode and polarization-insensitive structures in silicon-on-insulators and the coupling from submicrometric waveguides to single-mode fiber for optical telecommunication applications. Since 2003 he has been with the National Council for Scientific Research (CNRS), IEF, where his activities are related to passive (waveguide, splitters, $90^{\circ}$ turns, and optical couplers) and active (Si-based optical modulators and Ge-on-Si photodetectors) micro- and nanophotonic devices on silicon for optical interconnects and optical fiber communications. Since 2006, he has been in charge of the group of micro- and nanophotonic devices on silicon at IEF.

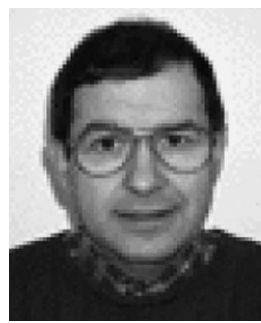

Daniel Pascal was born in Privas, France, in 1946. He received the Doctorat d'Etat degree in physics from the University Paris-Sud, Orsay, France, in 1981.

Since 1974, he has been with CNRS, Institut d'Electronique Fondamentale, Universite Paris-Sud, Orsay Cedex, France. He has been involved in Josephson magnetometry and GaAs photoconductivity. His current research interests include the field of silicon optoelectronic integrated circuits.

Sebastien Lardenois, photograph and biography not available at the time of publication.

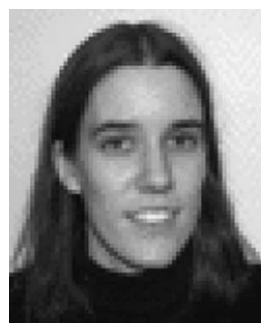

Delphine Marris-Morini received the Ph.D. degree from the University of Paris XI, Orsay Cedex, France, in 2004. Her Ph.D. thesis was on the design and realization of fast optical modulators integrated in silicon-on-insulator waveguides.

She is currently an Assistant Professor at the University of Paris XI, where she develops her research activity on silicon microphotonics.

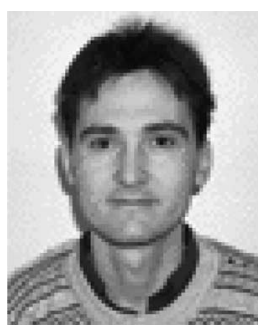

Eric Cassan received the Agrégation de Physique Appliquée and Ph.D. degrees from the Ecole Normale Supérieure de Cachan, Cachan Cedex, France, in 1995 and 2000, respectively.

He then joined the Institut d'Electronique Fondamentale, Universite Paris-Sud, Orsay Cedex, France. His interests are related to active optoelectronic components (photodetectors and modulators), passive photonic devices with sub-micrometric siliconon-insulator (SOI) waveguides, and SOI photonic crystals. 


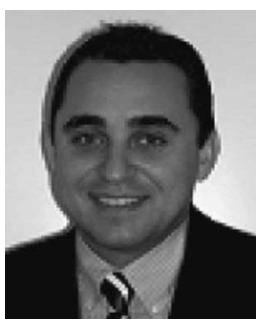

Frédéric Grillot (S'02-A'03) was born in Versailles, France, on August 22, 1974. He received the M.Sc. degree in physics on light-matter interaction from the University of Dijon, Dijon Cedex, France, in 1999 and the Ph.D. degree in electrical engineering from the University of Besançon, Besançon Cedex, France, in 2003.

His doctoral research activities were conducted within Opto+, Alcatel Research, and Innovation, Marcoussis, France. During his Ph.D. work, he studied optical feedback effects in semiconductor lasers. More particularly, he was in charge of the realization of feedback-resistant monomode DFB lasers for $2.5-\mathrm{Gb} / \mathrm{s}$ isolator-free transmissions. His work has led to understand both quantitatively and qualitatively the effects induced by optical feedback on the lasers and to relate these effects to the degradations in the bit error rate plots. Successful and state-of-the art $2.5-\mathrm{Gb} / \mathrm{s} 85^{\circ} \mathrm{C}$ transmissions without floor and with low penalties have been achieved, opening the way for all-optical telecommunication networks. From June 2003 to September 2004, he was with the Institut d'Electronique Fondamentale, Universite ParisSud, Orsay Cedex, France, through a postdoctoral position supported by Alcatel and the Centre National de la Recherche Scientifique, where his main research activities were on polarization-independent waveguides for telecommunication wavelengths and integrated optics modeling. Part of his work was devoted to calculate scattering loss induced by sidewall roughness within ultrasmall silicon-on-insulator optical waveguides. Since September 1, 2004, he has been a Lecturer at the Institut National des Sciences Appliquées, Rennes, France. His research activities are on quantum dot lasers and semiconductor optical amplifiers for optical telecommunications and low-cost applications.

Dr. Grillot is a member of IEEE-LEOS and la Société Française d'Optique.

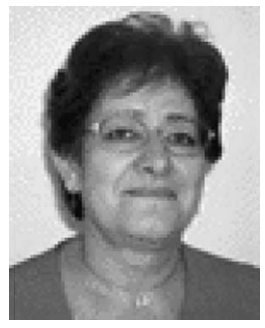

Suzanne Laval received the Doctorat es Sciences degree from the Institut d'Electronique Fondamentale, Universite Paris-Sud, Orsay Cedex, France, in 1973.

In 1967, she joined the CNRS, Universite ParisSud. Her first research work concerned nonlinear optics and IR emission from polaritons in crystals. She then experimentally demonstrated ballistic electronic transport in sub-micrometer electronic devices by photoconduction measurements. She has been involved in the first developments of optical bistability, optical switching, and optical logic devices. For several years, she has been concerned with integrated optics and optoelectronic devices on silicon-insulator (SOI) substrates. The first devices that have been realized are modulator arrays to form a spatial light modulator. The main application that she is currently aimed at is optical interconnects in microelectronics integrated circuits using technological processes compatible with the microelectronics ones on SOI substrates. Various kinds of devices for optical telecommunications will also be considered on the same SOI substrates.
Jean-Marc Fédéli received the Diploma in electronics engineering from INPG, Grenoble, France, in 1978

He was CEA-LETI, Grenoble, where developed different magnetic memories and magnetic components as Project Leader, Group Leader, and Program Manager. For two years, he acted as Advanced Program Director with the Memscap Company, developing RF-MEMS. In 2002, he returned to CEALETI as Coordinator of silicon photonic projects. Under a larger partnership with universities and research laboratories, he works on various technological aspects on photonics on CMOS ( $\mathrm{Si}$ rib and stripe waveguides, $\mathrm{Si}_{3} \mathrm{~N}_{4}$ and a$\mathrm{Si}$ waveguides), SiGe modulators, Ge photodetectors, Ge dots sources, and InP sources. His main focus is on the integration of a photonic layer at the metallization of an electronic circuit.

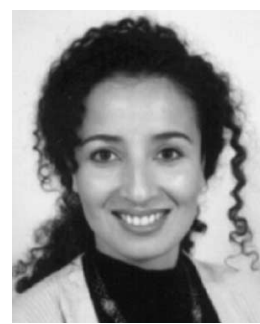

Loubna El Melhaoui received the Diploma in physics measurements technology from Grenoble University, Grenoble, France, in 2000.

Before joining CEA-LETI, Grenoble, she was with the Lithography Group, ATMEL, for one year. She works on all aspects of $\mathrm{SiC}$ technology for the development of power diodes. She is now involved with the characterization of silicon photonics components. Her main activity is focused on germanium technology and silicon modulator analysis. 